\title{
Insight of Artificial Intelligence Application in Healthcare
}

\author{
Cedric Kuang ${ }^{1}$
}

\begin{abstract}
${ }^{1}$ Zhuhai First Middle School, 2180 Meihua West Road, Xiangzhou District, Zhuhai City, Guangdong Province, China
\end{abstract}

\begin{abstract}
Ever since "Deep Blue" defeated the human champion chess player in 1997, artificial intelligence has caused extensive attention and discussion. With the development of computing and data storage technologies, artificial intelligence has been made great progress and applied in a wide range of fields. Here, we focus on several creative and novel applications of artificial intelligence in the healthcare field and also discuss their future trends.
\end{abstract}

\section{Introduction}

As the technology and economy develop rapidly in recent decades, healthcare problems have also made great progress. Traditionally, the only way to diagnose and cure diseases is through the knowledge and experience that controlled by clinicians, which is an ineffective way with high costs and error rates ${ }^{1-3}$. However, with the assistant of intelligence algorithms and accumulating data, artificial intelligence (AI) offers a number of advantages over traditional analytics and clinical decision-making techniques, such as diagnostics, care processes, treatment variability, patient outcomes and so on ${ }^{2}$. Here, we reviewed some applications that have already revolutionized in the field of healthcare. In addition to their current status, their future trends will also be discussed.

2. Specific Applications: AI has already successfully applied in a variety of medical fields, such as oncology, cardiology, gastroenterology, etc. ${ }^{4,5}$ In order to obtain a more comprehensive understanding in this topic, it is necessary to find out their current status and future trends.

2.1 AI in Managing Medical Data: Medical data, including treatments given, providers used, billed amounts, prescriptions, and so on, has been increased rapidly in recent years ${ }^{3}$. The main defect of these data is that they are not capable of providing adequate patients' health status and clinical severity ${ }^{4}$. To solve these problems, recently, these data are often combined with other data sources, such as census data and electronic medical records (EMRs) ${ }^{5}$. After combination, these data are able to provide more detailed patients' situations and information, including videos, photos, basic physiological indices and so on. However, some problems like massive and compilated data calculation and interpretation still remain unsolved. The high demand of standard statistical analysis ability presents challenges for clinicians ${ }^{3}$. Due to the ability of learning and extracting complex patterns from raw data, AI can play an important role in managing medical data ${ }^{6,7}$. For instance, machine learning can diagnose diseases by learning form experience (see Fig. 1a) and neutral network can find relation between two different pieces of information (see Fig. 1b) ${ }^{8}$.

Furthermore, AI has potentials to reduce the bias in healthcare when it manages medical records, including doctor biases (see Fig. 1c) and omitted variables biases $^{3}$. Doctor biases come from limitation of diagnosis time, shortage of information, personal experience, and forecasting biases ${ }^{9}$. AI copes with these shortcomings by combining more knowledge and varied experience to form a diagnosis tool, which can complement doctors' judgment with unbiased tool ${ }^{10,11}$.

To sum up briefly, medical records and data are important to both patients and doctors. AI can reduce mistakes and improve efficiency and utility in this field It can excavate much more useful information and draw conclusion that human may ignore. In the future, the application of AI in managing medical data will be prevailing and adopted by more and more hospitals since it provides fundamental assistance in medicine. 

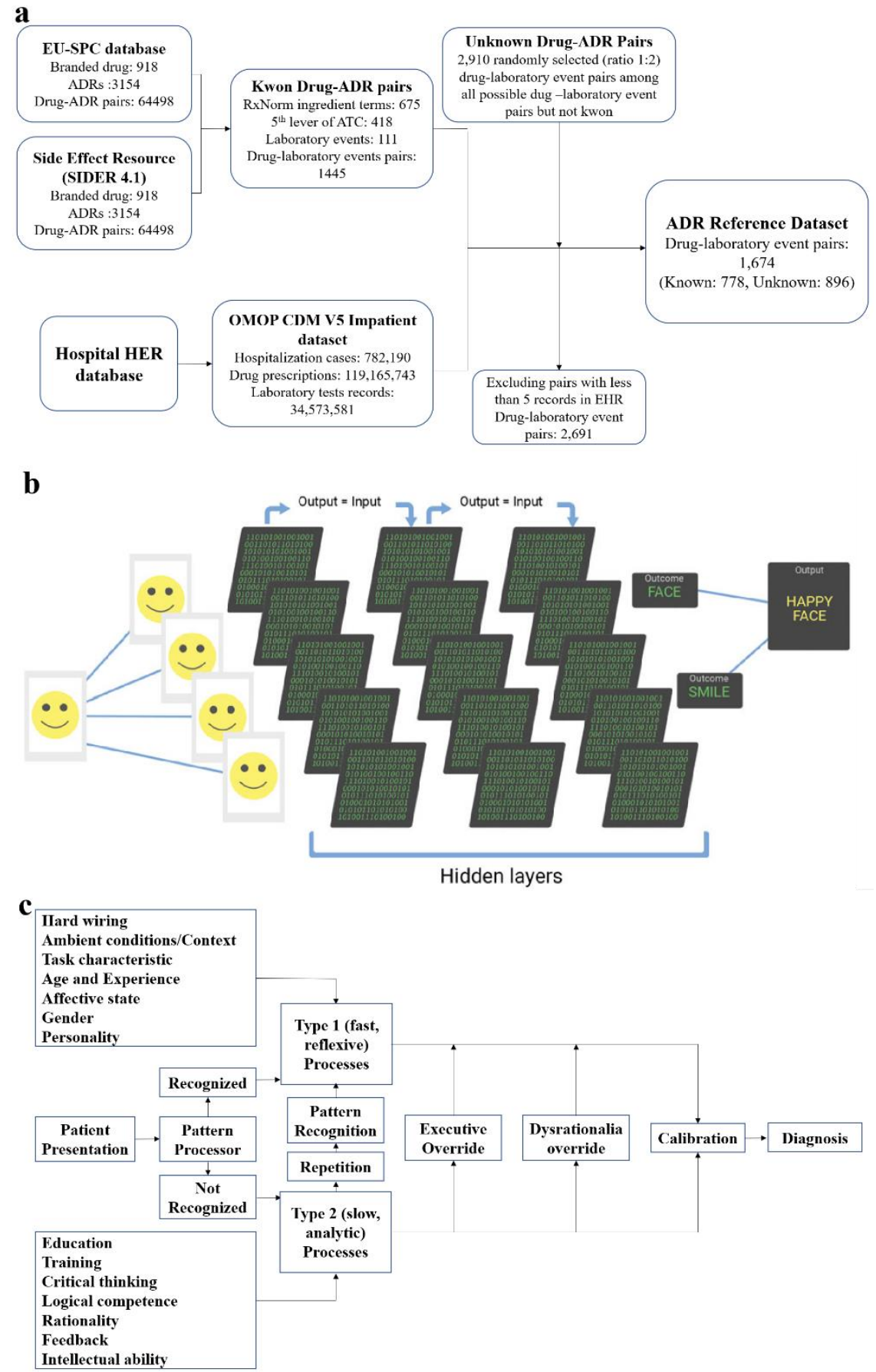

Figure 1. Machine learning ${ }^{6}$ (a) and neutral network ${ }^{1}$ (b) in managing medical data. (c) Effects of doctors' biases in the final diagnosis?

2.2 GeoAI in Healthcare: Geospatial science artificial intelligence (GeoAI) is the combination of methods in spatial science (e.g., geography information system or GIS), data mining and computer algorithms to extract data from raw information ${ }^{12}$.

GeoAI is meant to promote healthcare at the population level $^{13}$. First, GeoAI can improve environment health by detecting environmental exposure, some of which is difficult to capture by human ${ }^{12}$. Second, GeoAI can assist researchers to map and monitor the spatial spread of epidemics and to analyze their causes and consequences with geospatial data. Third, machine learning has potential to analyze DNA's and RNA's sequence of basic groups in gene-environment interaction (GxE) studies and to examine the intersections of genetics and environment, which offer an opportunity to restrict the spread of epidemics of eliminate them fundamentally ${ }^{14}$. Last but not the least, combine with EMRs, GeoAI is able to identify social and behavioral determinants of health and conduct interventions using geospatial data ${ }^{13}$.

In private sector, GeoAI is powerful in precision medicine as well, bases on detailed and enormous information about a patient's health. Precision 
medicine is a method to customize patients' treatment and diagnosis strategies through considering individual genetics, lifestyles and environment ${ }^{15}$. This technique is widely accepted in mobile health applications which provides convenient and basic self-diagnosis. By referring to patients' history, these applications can provide recommendations in treatments and conclude the environmental factors ${ }^{16}$. A snapshot and a schematic of relations of different factors in GeoAI are shown in Fig. 2.

In conclusion, GeoAI acts as an important role in both public and private healthcare. It turns geospatial data into practice, placing the whole society as beneficiary. In the future, GeoAI will enter more and more fields in research and daily life.

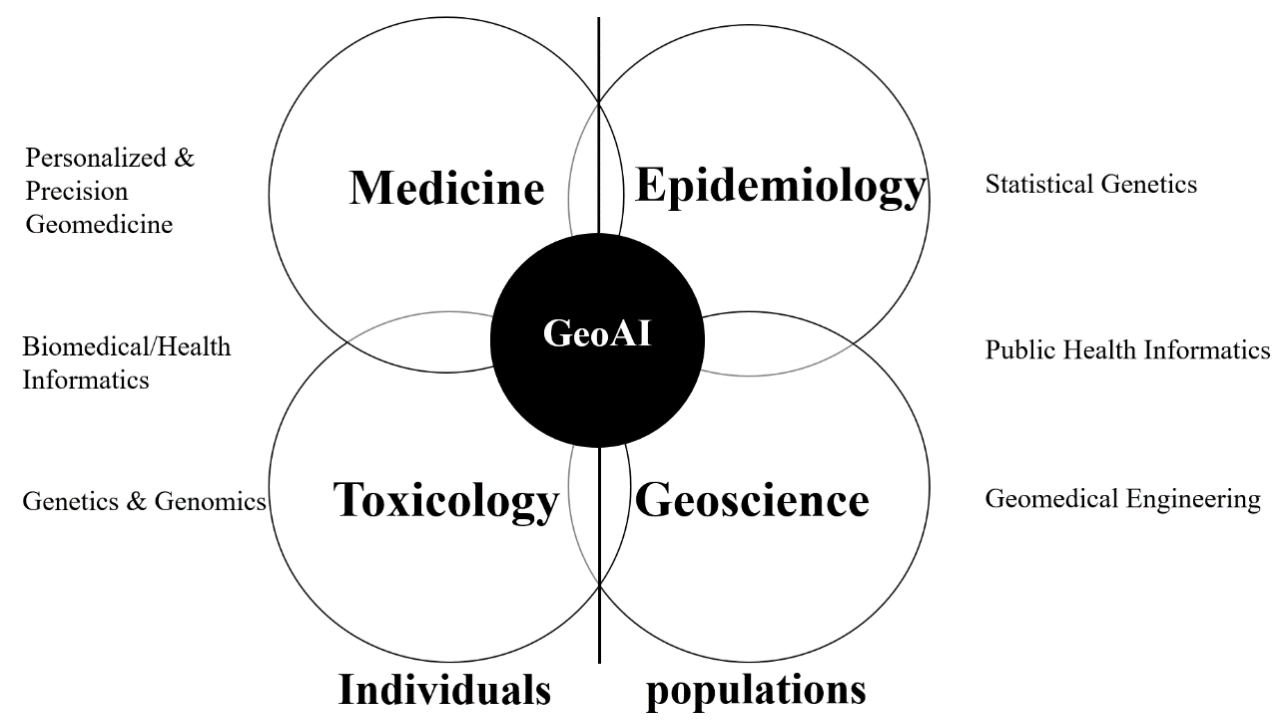

Figure 2. Different factors in GeoAI ${ }^{16}$.

2.3 AI in Mental Health: In mental health domain, AI is also a powerful diagnosis tool. One of the main applications is monitoring. AI can record patients' mood diaries, sleeping patterns and some other physiological conditions, even outside the clinical setting. Adequate monitoring benefits (potential) patients a lot, especially in the early detection and prevention of relapse, which have significant impacts on outcomes ${ }^{17}$. Sensors can collect multiple types of data such as distance travelled, variation of voice, speaking rate and voice quality, which are good predictors of symptoms of depression and post-traumatic stress disorder (PTSD) ${ }^{18}$. Another aspect of monitoring is medicine adherence, causing a big issue in medicine quality and allergic reactions. Machine learning is able to analyze patients' history to draw a conclusion about the effect of a particular medicine and allergen, which can facilitate the quality and customization of treatments ${ }^{19}$.

Furthermore, basing on machine learning and voice recognition, AI chatbots can directly assist clinicians in mental disease treatments. According to a research, college students who have conversations with chatbots decrease their depressions and anxiety ${ }^{20}$.

Generally speaking, AI in mental healthcare can drastically reduce the burden of clinicians and therapists, especially in complicated and monotonous chores. After the re-balance of workload, clinicians can pay more attention to the interactions with patients, which improve the quality of treatments ${ }^{17}$. The future prospect of AI in mental healthcare will be promising, since the number of people who pursue mental health increases swiftly these years.

2.4 AI in Surgery: Surgery is the most technique-needed and complicated process in treatment and AI can improve many aspects in surgery operations, such as anesthesia support, time management and patient's safety, monitoring patient's physiological indices, etc. ${ }^{21}$. Due to the characteristics of machine learning, the more surgery it processes, the more experience it can learn, resulting in improvement in efficiency and safety. Therefore, the value of AI in surgery is to improve accuracy and safety, reduce the burden of operator, and even enable automatic operations ${ }^{1}$.

In conclusion, since operation is a vital procedure in many treatments, both clinicians and patients welcome the benefits brought by AI. AI can assist a surgeon not only by enhancing motor skills, but also augment the surgeon's thought process and knowledge, which will improve patient outcome. The future combination of surgery and AI will be more promising and effective.

2.5 AI in Radiology: In recent years, AI has been applied to radiology in some aspects. Especially for deep-learning based radiology, the effectiveness of imaging is improved $^{22}$. Deep learning is suitable for image analysis and experience comprehension. Therefore, when it comes to multi-layer image, AI can convert the complication into simplicity. The inception 
layer is form by a combination of multiple convolution filters, which helps in quick convergence and generalization without increasing the complexity ${ }^{1,22}$. Figure $3 \mathrm{~A}$ gives an example of inception layer in detection of fractures and calculation of the trabecular bone density. Deep learning is also widely applied in treatment in MR-based ischemic stroke ${ }^{23}$ and CT-based respiratory disease ${ }^{24}$. A deep learning model with three layers (Layer 1 output: arrow (a); Layer 2 output: arrow (b) Layer 3 output: arrow (c)) is shown in figure 3B. Moreover, due to the cost of computer hardware and software decreases these years and the performance keeps going higher, it drastically decreases the time and budget for hospital to utilize and train the deep-learning-based radiology system ${ }^{22,25}$.

Furthermore, especially in radiology domain, the traditional methods may be ineffective in the future. With the support of AI technology, the effectiveness can be guaranteed. A radiologist can analyze an average 200 case a day ${ }^{26}$. However, a deep-learning-based radiology system can help radiologists to improve efficiency and precision by reading 600 cases a day, both remotely and locally ${ }^{22}$. Moreover, the combination of artificial and human intelligence can result in better prediction. As for stable and continuous data, deep learning can perform its powerful functions to the greatest extent. Whereas, if the data is sporadic or the quality is low, the hybridization of human and computer can offer better accuracy $^{27,28}$.

In conclusion, AI is able to manage most of the complicated and repeated work in radiology. However, clinicians are still the decision makers and monitors. The basic effect of AI in radiology is the enhancement of efficiency and accuracy. Besides, it can offer a better connection between patients and doctors.
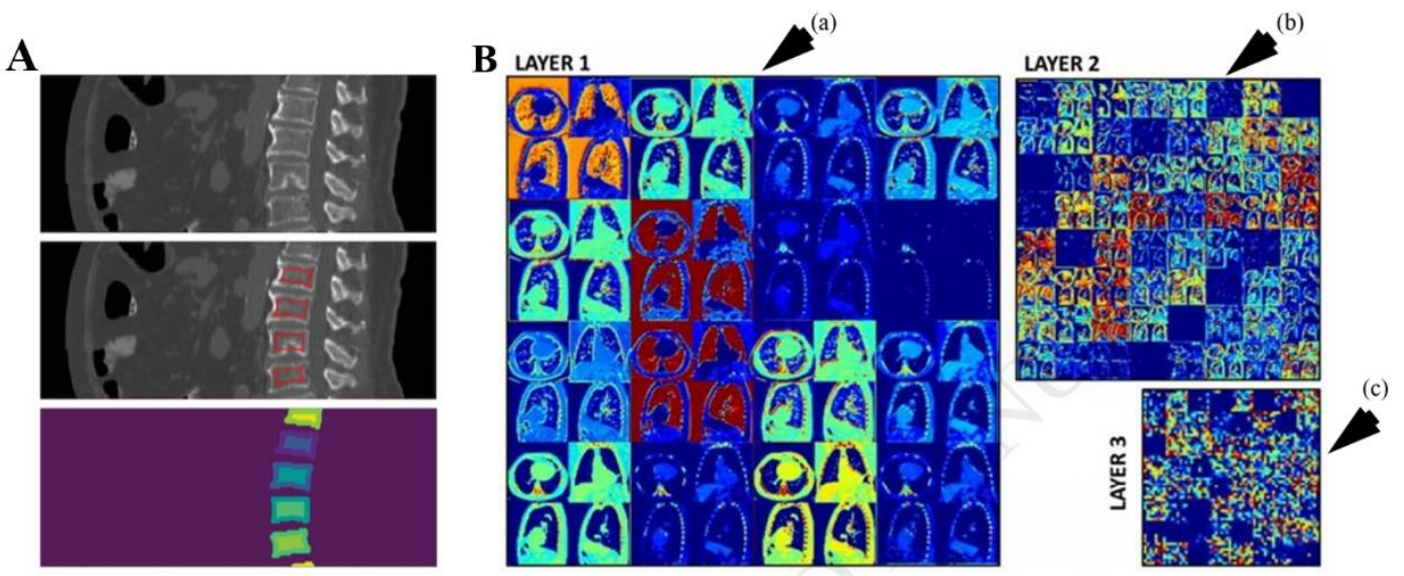

Figure 3. Inception layer ${ }^{1}$ (A) and deep learning model (B) in radiology $y^{22}$.

2.6 AI in Personalized Treatment: Molecular biomarkers and pharmacogenetic tools are able to demonstrate and predict drug efficiency and treatment response in patients. These components play a vital role in development of precision medicine ${ }^{29}$. Previously, identifying and developing a new treatment option is extremely challenging. However, AI can manage this job by using deep learning. AI is capable of identifying genetic variants and molecular factors by using data mining and transforming the harvest evidence and assays' results into real practice ${ }^{30}$. These are integrated into a deep learning model shown in Figure 4. Therefore, the tailored treatments offered by AI can result in better outcome in particular diseases than unified treatments do. In the future, personalized treatment will be a major trend in healthcare, and AI will be the most effective and useful treatments provider.

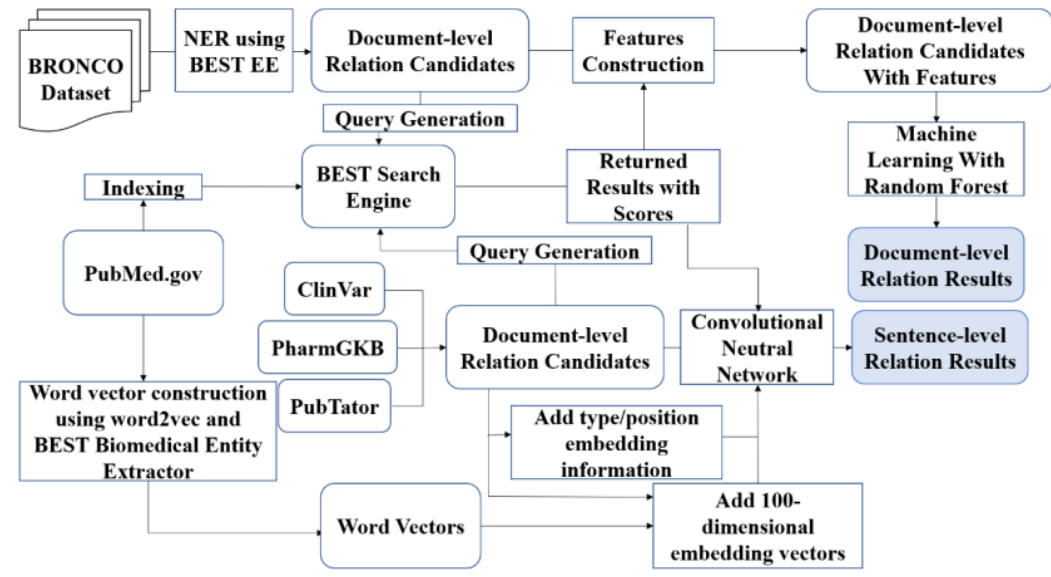

Figure 4. Deep learning models in precision medicine ${ }^{30}$. 
2.7 AI in Ophthalmology: AI in ophthalmology has been well-cooperated to diseases such as retinopathy of prematurity (ROP), diabetic retinopathy, glaucoma, etc. $^{31}$. Two main types of technique can manage this job well- machine learning and natural language processing, which is a method that extracts information from raw data and converts them into data that can be analyze by machine learning. With the support of remote screening technique, $\mathrm{AI}$ is able to detect eye diseases in their early stages for the people living in rural areas. Moreover, AI adapted in smartphones plus basic ophthalmology equipment allow diagnosis to expand beyond the confines of clinic offices ${ }^{32}$. In other words, AI can assist the management of patients both in office and those in remote areas as well.
Specifically, diabetic retinopathy (DR) affects millions of people's normal function of retina, resulting in reduction of vision or even fully blindness ${ }^{33}$. According to a study, AI had a higher sensitivity and specificity rate in analyzing patients' data and images than seven experts in ophthalmology $\mathrm{did}^{34}$, which proves that AI has significant potential in the early diagnosis of DR. As shown in Figure 5, two types of validation sets are applied to detect DR with the support of AI.

Generally speaking, as the population who are possessed with optical diseases increases exponentially these years due to the excessive usage of smart devices, AI will play a key role in the diagnosis of ophthalmic diseases.

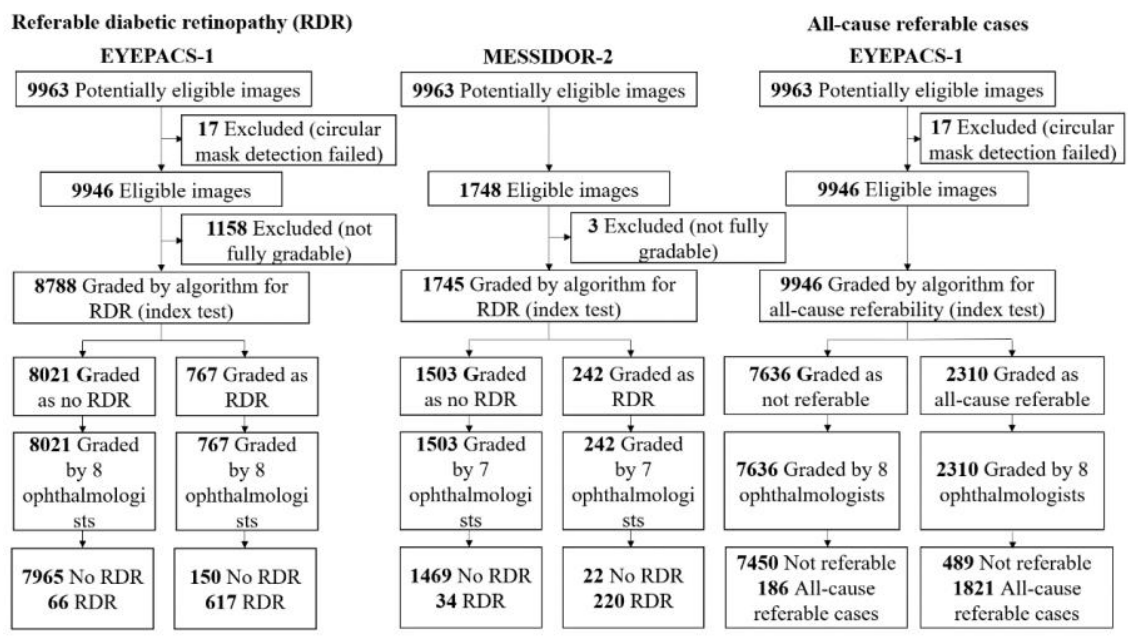

Figure 5. Two types of clinical validation set for detection of diabetic retinopathy ${ }^{34}$.

3. Risks and challenges: Even though AI can offer many advantages in many fields, it still has several defects and risks remained to be solved. First, just like most of the computer programs, bugs are still unavoidable as well in AI. Indeed, these bugs may bring serious damage to patients and clinicians. Second, AI is an algorithm after all, which needs an artificial database to train itself. This required private server to store patients' data, increasing the probability of data breach. Third, it augments people's cautiousness that AI in medicine is something related to their health. When it comes to health problems, people tend to rely more on doctors instead of a cold machine. Human interactions play a necessary role in treatment. Therefore, AI is not meant to and definitely cannot replace human clinicians, but rather act as an assistance to improve the abilities of clinicians. The final decision makers should be the clinicians.

4. Conclusion: AI is a widely applied technology in many fields, which is able to improve the efficiency and precision and to reduce the cost. In healthcare domains, the main jobs of AI are pre-diagnosis and assisting treatments. The integration of $\mathrm{AI}$ and healthcare data has significantly improved the workflow, meanwhile, decreased heavily the burden of clinicians. GeoAI is able to improve health from public and private view. AI in surgery, radiology and ophthalmology enhances the precision and accuracy of diagnosis. AI is a perfect assisting tool in curing mental diseases and offering personalized treatments as well. While the potential seems to be practical, some of them still remains theoretical and exists some risks and challenges, which requires further research and practice. In conclusion, the future of $\mathrm{AI}$ in healthcare is promising, but it has a long journey to go.

\section{Reference}

1. Mintz Y, Brodie R. Introduction to artificial intelligence in medicine. Minim Invasive. Ther. Allied. Technol. 2019, 28, 73 81.

2. Yokota H, Goto M, Bamba C, et al. Reading Efficiency Can Be Improved by Minor Modification of Assigned Duties: Pilot Study on a Small Team of General Radiologists. Jpn. J. Radiol. 2017, 35, 262-268.

3. Thesmar D, Sraer D, Pinheiro L, Dadson N, Veliche R, Greenberg P. Combining the Power of Artificial Intelligence with the Richness of Healthcare Claims Data: Opportunities and Challenges. Pharmacoeconomics. 2019, 37, 745-752.

4. Birnbaum HG, Cremieux PY, Greenberg PE, LeLorier J, Ostrander J, Venditti L. Using Healthcare Claims Data for Outcomes Research and Pharmacoeconomic Analysis. Pharmacoeconomics. 1999, 16, 1-8.

5. Cadarette SM, Wong L. An Introduction to Health Care Administrative Data. Can. J. Hosp. Pharm. 2015, 68, 232237.

6. Jeong E, Park N, Choi Y, Park RW, Yoon D. Machine Learning Model Combining Features From Algorithms with Different Analytical Methodologies to Detect Laboratory-event-related Adverse Drug Reaction Signals. PLoS. One. 2018, 13, 1-15.

7. Tulabandhula T, Rudin C. On Combining Machine Learning 
with Decision Making. Machine Learning. 2014, 97, 33-64.

8. LeCun Y, Bengio Y, Hinton, G . Deep Learning. Nature. 2015, 521, 436-444.

9. Saposnik G, Redelmeier D, Ruff CC, Tobler PN. Cognitive Biases Associated with Medical Decisions: a Systematic Review. BMC. Med. Inform. Decis. Mak. 2016, 16, 1-14.

10. Gibert K, Garcia-Alonso C, Salvador-Carulla L. Integrating Clinicians, Knowledge and Data: Expert-based Cooperative Analysis in Healthcare Decision Support. Health Res. Policy Syst. 2010, 8, 1-16.

11. Seidman AD, Pilewskie ML, Robson ME, Kelvin JF, Zauderer MG, Epstein AE, et al. Integration of Multi-modality Treatment Planning For Early Stage Breast Cancer (BC) into Watson for Oncology, a Decision Support System: Seeing the Forest and the Trees. J. Clin. Oncol. 2015, 33.

12. VoPham T, Hart JE, Laden F, Chiang YY. Emerging Trends in Geospatial Artificial Intelligence (GeoAI): Potential Applications for Environmental Epidemiology. Environ. Health. 2018, 17, 40.

13. Boulos MK, Peng G, VoPham T. An Overview of GeoAI Applications in Health and Healthcare. Int. J. Health Geogr 2019, 18, 7.

14. McAllister K, Mechanic LE, Amos C, Aschard H, Blair IA, Chatterjee N, Conti D, Gauderman WJ, Hsu L, Hutter CM, et al. Current Challenges and New Opportunities for Gene-environment Interaction Studies of Complex Diseases. Am. J. Epidemiol. 2017, 186, $753-761$.

15. Davis MM, Shanley TP. The Missing-Omes: Proposing Socia and Environmental Nomenclature in Precision Medicine. Clin. Transl. Sci. 2017, 10, 64-66.

16. Boulos MN, Le Blond J. On the Road to Personalised and Precision Geomedicine: Medical Geology and a Renewed Call for Interdisciplinarity. Int. J. Health Geogr. 2016, 15, 5.

17. Lovejoy C, Buch V, Maruthappu M. Technology and Mental Health: The Role of Artificial Intelligence. Eur. Psychiatry. 2018, 55, 1-3.

18. Place S, Blanch-Hartigan D, Rubin C, Gorrostieta C, Mead C, Kane J, Marx B, Feast J, Deckersbach T, Pentland A Nierenberg A, Azarbayejani A. Behavioral Indicators on a Mobile Sensing Platform Predict Clinically Validated Psychiatric Symptoms of Mood and Anxiety Disorders. J. Med. Internet Res. 2017, 19,3

19. Stentzel U, Van den Berg N, Schulze LN, Schwaneberg T, Radicke F, Langosch JM, Et al. Predictors of Medication Adherence Among Patients with Severe Psychiatric Disorders: Findings from the Baseline Assessment of a Randomized Controlled Trial (Tecla). BMC Psychiatry 2018, 18, 155.

20. Fitzpatrick KK, Darcy A, Vierhile M. Delivering Cognitive Behavior Therapy to Young Adults with Symptoms of Depression and Anxiety Using a Fully Automated Conversational Agent (Woebot): A Randomized Controlled Trial. JMIR Ment. Health. 2017, 4, 2 .
21. Rockstroh $M$, Franke S, Hofer $M$, et al. OR.NET: Multi-perspective Qualitative Evaluation of an Integrated Operating Room Based on IEEE 11073 SDC. Int. J. CARS. 2017, 12, 1461-1469.

22. Saba L, Biswas M, Kuppili V, Cuadrado Godia E, Suri HS Edla DR, Omerzu T, Laird JR, Khanna NN, Mavrogeni S, Protogerou A, Sfikakis PP, Viswanathan V, Kitas GD, Nicolaides A, Gupta A, Suri JS. The Present and Future of Deep Learning in Radiology. Eur. J. Radiol. 2019, 114, 14-24.

23. Nielsen A, Hansen MB, Tietze A, Mouridsen K. Prediction of Tissue Outcome and Assessment of Treatment Effect in Acute Ischemic Stroke Using Deep Learning. Stroke. 2019, 6, 1394 1401 .

24. González G, Ash SY, Vegas-Sánchez-Ferrero G, Onieva JO, Rahaghi FN, Ross JC, Díaz A, Estépar RSJ, Washko GR. Disease Staging and Prognosis in Smokers Using Deep Learning in Chest Computed Tomography. Am. J. Resp. Crit. Care. 2018, 2, 193-203.

25. Barham P, Chen J, Chen Z, Davis A, Dean J, Devin M, et al. TensorFlow: A System for Large-Scale Machine Learning. In. OSDI. 2016, 16, 265-283.

26. IBM Research Accelerating Discovery: Medical Image Analytics, 10/10/2013. Available Online. https://www.youtube.com/watch?v=0i11 VCNacAE

27. Liew C. The Future of Radiology Augmented with Artificial Intelligence: A Strategy for Success. Eur. J. Radiol. 2018, 102, 152-156.

28. Nagar, Yiftach, Malone TW, De Boer P, Garcia ACB. Essays on Collective Intelligence. $\mathrm{PhD}$ diss., Massachusetts Institute of Technology. 2016

29. Gruson D, Helleputte T, Rousseau P, et al., Data Science, Artificial Intelligence, and Machine Learning: Opportunities for Laboratory Medicine and the Value of Positive Regulation. Clin. Biochem. 2019, 69, 1-7

30. Lee K, Kim B, Choi Y, Kim S, Shin W, Lee S, et al. Deep Learning of Mutation-Genedrug Relations from the Literature. BMC Bioinformatics [Internet]. 2018, 19, 21. Available from: https://bmcbioinformatics.biomedcentral.com/articles/10.1186/ s12859-018-2029-1

31. Rathi S, Tsui E, Mehta N. The Current State of Teleophthalmology in the United States. Ophthalmology. 2017 , 124, 1729-1734.

32. Kappor R, Walters SP, Al-Aswad LA, The Current State of Artificial Intelligence in Ophthalmology. Surv. Ophthalmol. $2018,64,233$

33. Organization WH. Global Report on Diabetes 2016. Available from: http://www.who.int/diabetes/globalreport/en/.

34. Gulshan V, Peng L, Voram M, et al. Development and Validation of a Deep Learning Algorithm for Detection of Diabetic Retinopathy in Retinal Fundus Photographs. JAMA. 2016, 316, 2402-2410. 